\title{
Nueva piel para una vieja ceremonia. Una mirada al Concurso Centro Cultural Gabriela Mistral
}

\section{De arquitecturas públicas y concursos}

Para nadie es un misterio que los concursos abiertos constituyen una de las mejores oportunidades para obtener y asegurar la calidad en una obra de arquitectura, especialmente en lo referido al ámbito público. No podemos dejar de argumentar que aquellos lugares que muestran un desarrollo del quehacer arquitectónico más dinámico, corresponden a países que han sostenido e impulsado un proceso continuo y sostenido de concursos, como instancias que permiten una reflexión intelectual sobre una temática determinada y por lo tanto, son capaces de configurar nuevos ensayos con respecto a las problemáticas contemporáneas relevantes en el espacio habitado. Un caso paradigmático lo constituye la situación española ${ }^{1}$

En el contexto de nuestro país, hemos de admitir que la tradición de concursos -que conlleva siempre una instancia democrática, anónima en su proceso, y que implica un juicio fundamentado y accesible-, ha estado un tanto ajena al desarrollo de la disciplina por un período de tiempo más largo del que quisiéramos, y de los eventos realizados, un número no menor ha tenido resultados accidentados e inciertos ${ }^{2}$. En el ámbito de la inversión pública, todavía llevamos a cuesta el lastre de un desvencijado sistema de licitaciones, que además de valorar la respuesta arquitectónica a la problemática abordada, asume también otras variables

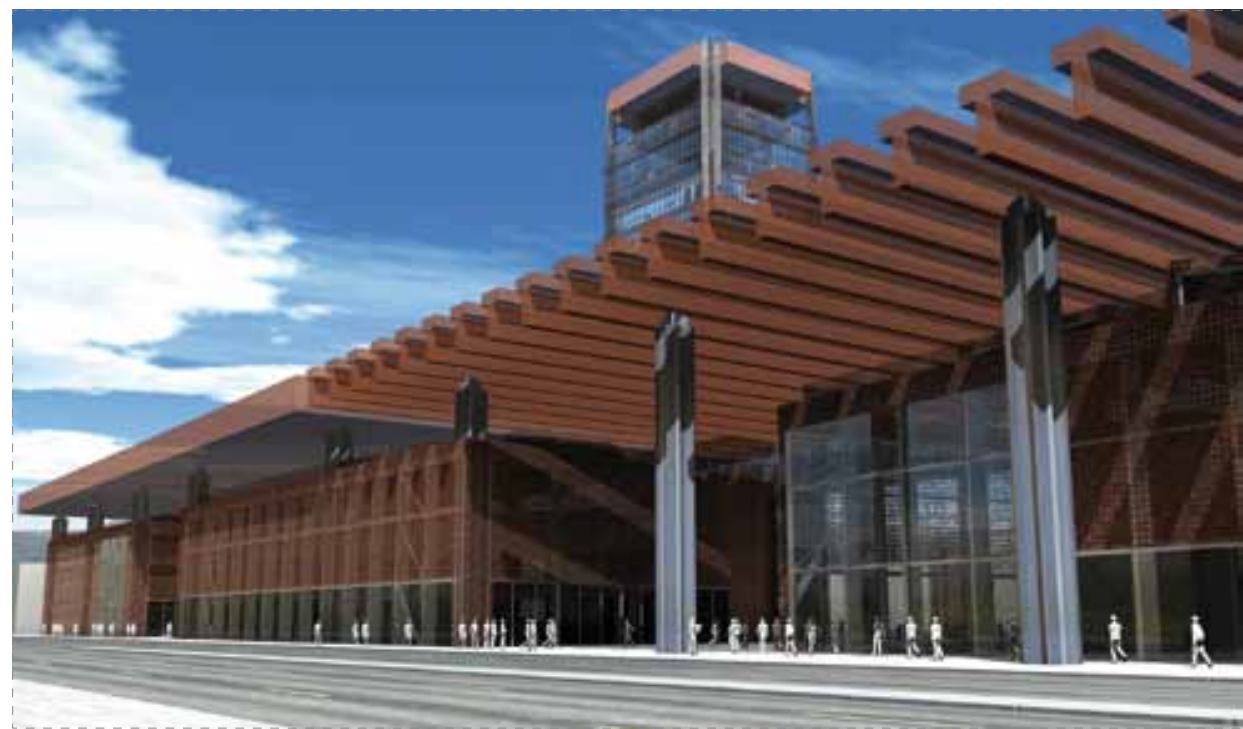

(como la oferta de honorarios profesionales, por ejemplo), en los factores de apreciación de la propuesta. Un punto de inflexión en este panorama corresponde al llamado realizado por la Dirección de Arquitectura del Ministerio de Obras Públicas a participar de concursos en una serie de proyectos de escala relevante para la Ciudad de Santiago durante el año 2007, entre los cuales se cuenta el Museo de la Memoria, emplazado en la fallida estación intermodal de Quinta Normal; el Edificio Corporativo de Correos de Chile, en el sector de Estación Mapocho; y el Nuevo Centro
Cultural Gabriela Mistral, ubicado en el Barrio Lastarria.

Con respecto a los concursos antes citados, una característica común lo constituye el hecho de que corresponden a proyectos de intervención en cascos históricos consolidados y lugares centrales, pero con algún grado de deterioro, abandono o simplemente en fricción desde el punto de vista de su funcionamiento e imagen urbana. Un hecho auspicioso, pues permite detectar una cierta sensibilidad que contribuye al ejercicio de completar a modo

Arquitecto, Universidad de Chile. Master en Arquitectura, Historia, Arte y Ciudad, Universitat Politécnica de Catalunya. Académico Universidad de Chile y Universidad de Santiago

De acuerdo a la legislación española, y las políticas vigentes para edificación pública, toda obra que se deba edificar y que implique un impacto en la ciudad debe ser objeto de un concurso público de arquitectura. En algunos casos, se incentiva la participación de jóvenes oficinas de arquitectura en concursos especiales como una manera de democratizar el acceso a la obtención de proyectos.

2 Al respecto, cabe ejemplificar con la polémica generada por el concurso para el nuevo Ministerio de Relaciones Exteriores en el año 2001, adjudicado al arquitecto Teodoro Fernández, que finalmente no se construye, pues el Gobierno decide ocupar las dependencias del Hotel Carrera. Ver Revista CA № 112, Santiago, 2003. Un ejemplo más reciente corresponde al errático desenlace del Concurso para la Ampliación del Museo de Bellas Artes. 


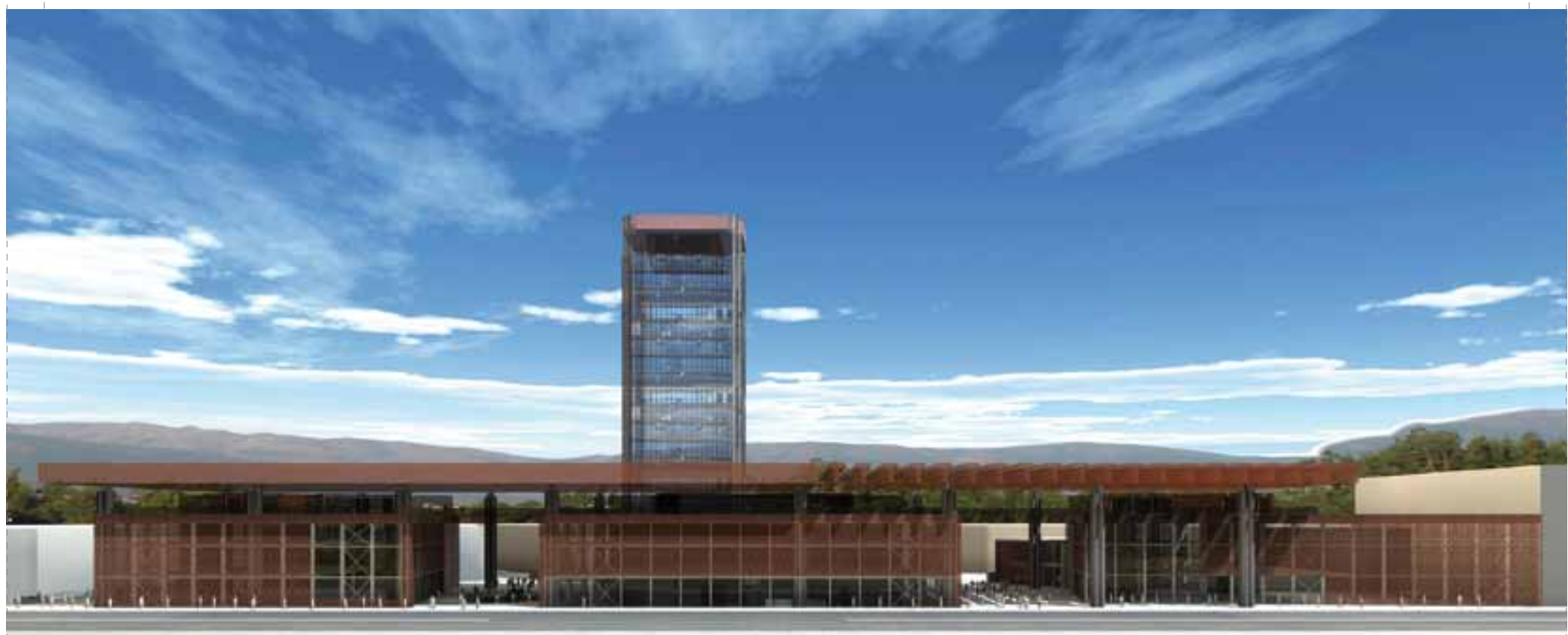

de sutura, el espacio urbano, plataforma imprescindible para el ejercicio de actividades que permiten la expresión de la ciudadanía.

En relación al Centro Cultural Gabriela Mistral, concurso del que nos ocuparemos más en detalle, es posible afirmar que la reconversión del edificio llega después de un largo proceso de reflexión y discusión en torno al destino que debía dársele, tras el incendio que destruyó el ala oriente, el 5 de marzo de 2005.

\section{Centro Cultural Gabriela Mistral: Pieles y suturas}

Construido originalmente entre 1971 y 1972, bajo el gobierno de Salvador Allende, el complejo fue diseñado por un grupo de arquitectos liderados por Juan Echeñique y Miguel Lawner para albergar en una primera instancia la Tercera Conferencia Mundial de Comercio y Desarrollo de las Naciones Unidas. Luego de realizado el evento, el edificio se destinaría a un centro cultural abierto y popular, un vehículo de expresión donde la sociedad pudiese reunirse en torno al cultivo de las artes. Sin embargo, en el momento en que comienza la reconversión del edificio como centro cultural, el proceso queda interrumpido por la caída del gobierno de la Unidad Popular, en septiembre de 1973. Con la llegada de la Junta Militar al poder, el promisorio centro queda destinado a las instalaciones del Ministerio de Defensa y como punto relevante de administración y toma de decisiones del poder político, adoptando el nombre de Diego Portales.

Una vez recuperada la Democracia, el edificio conservará su programa como Ministerio de Defensa, pero al mismo tiempo, comenzarán a hilvanarse discusiones aisladas, pero cada vez más persistentes sobre la pertinencia de recuperar la finalidad original del edificio como centro cultural ${ }^{3}$. El incendió registrado en 2005 probablemente actuará como detonante para encausar las discusiones y formalizar los esfuerzos tendientes a la recuperación programática antes mencionada que finalmente verá la luz con la convocatoria al Concurso, realizada durante el segundo semestre de 2007.

De acuerdo a lo señalado en las bases de concurso, programáticamente el edificio se debía concebir como un gran centro de artes escénicas y música, un espacio de formación audiovisual, de talleres, de ensayo. El proyecto también debería incluir espacios para centros de convenciones, conferencias y una biblioteca pública.
El proyecto ganador para el Nuevo Centro Cultural Gabriela Mistral, cuya autoría corresponde a los arquitectos de la Universidad de Chile, Cristián Fernández Eyzaguirre, en asociación con Sebastián Barahona y Christian Yutronic, intentará abordar la complejidad de la habilitación del edificio, incorporando y yuxtaponiendo la diversidad del programa planteado con una nueva trama de recorridos y espacios públicos para potenciar y consolidar los trasvasijes entre la Alameda -cuya fachada se configura como principal- y el interior del Barrio Lastarria. De esta manera, sería posible afirmar la hipótesis del reemplazo de la percepción del edificio como volumen obstruido por un cuerpo articulador con el contexto ya consolidado.

Si una de las principales críticas que se formulaban al antiguo edificio era la indiferencia hacia la escala peatonal, dada principalmente por la envergadura monumental de la placa, la estructura estereométrica de cubierta y la opacidad de la fachada, la apertura del edificio en el nivel principal, a partir de portales que actuarán como cauces e incentivarán el flujo, constituye a mi juicio uno de los méritos más relevantes de la propuesta, pues contribuirá a incentivar el traspaso e incorporar el interior de la manzana a la actividad citadina. Es posible

\footnotetext{
Como botón de muestra, resulta interesante revisar la polémica suscitada a partir del artículo sobre la reconstrucción del edificio Diego Portales publicado en el Suplemento Artes y Letras del diario El Mercurio. Santiago, 12 de agosto de 2007.
} 
afirmar además que la apertura señalada motivará un uso intensivo en el espacio público generado, toda vez que salas de música y teatros, cafés, librerías, talleres y espacios de exposición alentarán condiciones de flexibilidad de uso, relevantes en cuanto a acercar el futuro centro a la comunidad, uno de los objetivos declarados en las políticas vigentes sobre la creación de nuevas infraestructuras para la cultura, coincidiendo así dichos lineamientos con lo señalado por Luis Rojo, en cuanto a que «la flexibilidad, como noción asociada a la estrategia disciplinar, corre paralela a las aspiraciones de la cultura de masas, configurándose como uno de los instrumentos fundamentales para alcanzar la desinstitucionalización del museo y su substitución por un espacio cívico y democrático» ${ }^{4}$.

Desde otra perspectiva, el proyecto resalta el potencial del edificio existente, con una intervención respetuosa sin por ello alcanzar el nivel de réplica. Muy por el contrario, a través de un proceso selectivo y cuidadoso, se han conservado los pilares, como una forma de preservar la magnitud de la obra, algunas losas de primer y segundo nivel y la cubierta, que se reformula a partir de una nueva envolvente y se completa de manera más liviana en la zona dañada por el incendio, a partir de la ejecución de una serie de vigas permeables. De acuerdo a lo anteriormente señalado, en la reinterpretación de la placa se genera de forma más visible, una delicada articulación entre el pasado del edificio y su condición a futuro, proveyendo de un tratamiento armónico entre la edificación existente y la propuesta. Desde esta posición, el proyecto se sitúa en concordancia con lo señalado por Francisco de Gracia: «intervenir equivale a actuar conscientemente en el proceso dinámico de la ciudad; debiendo añadirse que, en todo caso, habría de garantizarse la mínima estabilidad necesaria para que la forma urbana, en sus partes y en el todo, prolongue una identidad que ha sido conseguida lenta y trabajosamente. La ciudad es un patrimonio del pasado a transferir hacia el futuro y, si es posible, mejorado por el presente» 5 .

En la misma línea, habría que agregar que la generación de seis plazas, en la zona de cauces o flujos y donde el edificio se encuentra con la trama de la ciudad consolidada

4 Luis Rojo de Castro. Dominando el domi-no. En: Circo, Boletín Técnico. Luis M. Mansilla + Luis Rojo + Emilio Tuñón Editores. Madrid, 2004; 120.

5 Francisco de Gracia. Construir en lo construido. arquitectura como modificación. Madrid: Editorial Nerea, 1992
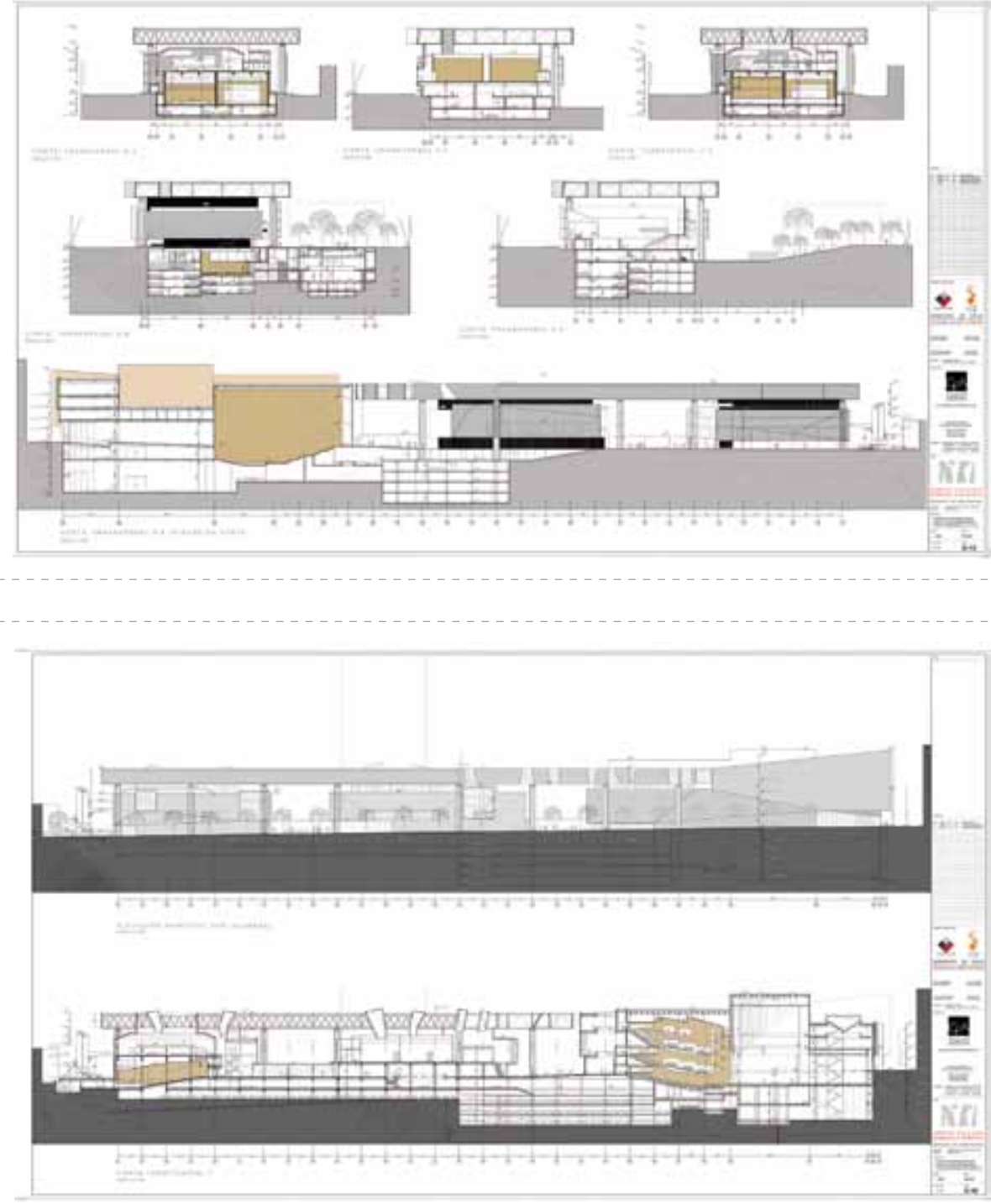

refuerza desde el punto de vista de los usos la idea de engarce entre las huellas de la historia y la nueva intervención, toda vez que serán éstas las encargadas de permitir una permeabilidad entre interior y exterior y una continuidad sin roces en el recorrido y la pausa del usuario, completando y develando las tramas existentes.

Desde el punto de vista de la imagen, resulta importante señalar que el proyecto de Fernández, Barahona y Yutronic no busca recrear de modo escenográfico condiciones preexistentes, situación que redunda en un tratamiento seductor, pero superficial de las cáscaras urbanas atrayendo al público usuarioconsumidor por medio de simulacros, tal como se aprecia en algunas intervenciones cercanas.
Un desafortunado caso que puede citarse es la operación inmobiliaria actualmente en desarrollo de calle Lastarria, la cual intenta atraer, pero al mismo tiempo ocultar por medio de una fachada histórica, la ocupación de la manzana por parte de un conjunto de edificios homogéneos, comprimidos y con una lectura ajena desde el punto de vista de la imagen urbana del sector. En oposición a ello, el proyecto para el Centro Cultural Gabriela Mistral intenta transformar cualitativamente el tejido urbano, a partir de la agregación de una piel contemporánea en un material pregnante como el acero perforado, que se adapta y cubre las fachadas del edificio, resignificándolas, sugiriendo su estructura original y mejorando la escala del edificio, especialmente en su cara principal hacia la 
Alameda. De esta forma, es posible afirmar que la intervención propuesta para el centro será capaz de transformar cualitativamente el tejido histórico del contexto en el cual se inserta, regenerándolo a través de una nueva membrana y revitalizándolo con una perspectiva contemporánea.

\section{Consideraciones al margen}

Es de esperar que el actual proyecto -actualmente en pleno proceso de diseño-, logre sortear las complejas coordinaciones y requerimientos técnicos que una obra de tal complejidad siempre trae consigo. No olvidemos que en el proyecto están involucrados varios actores relevantes, entre los que se cuenta no sólo el Ministerio de Obras Públicas, sino además el Consejo de la Cultura y las Artes y el Ministerio de Defensa. De ello depende en parte el éxito de una tarea que se advierte provechosa para amalgamar un barrio valorado de la ciudad, enriquecedora como soporte cultural metropolitano y que finalmente intentará completar el guión -con una lectura contemporánea-, de una historia que comenzó hace cerca de cuatro décadas y que no se extinguió con aquel incendio de marzo de 2005.

Finalmente, una vez que el edificio entre en funciones, necesitaremos confiar en la enorme gestión cultural que se deberá realizar, para que el nuevo Centro Cultural Gabriel Mistral sea un motor activo, eficaz y dinámico, y no se convierta, haciendo uso de las reflexiones de Iñaki Esteban al referirse a la pérdida de funciones de un espacio museístico, «en una especie de adorno con una decoración cultural sin fuerza para cumplir con la trascendencia social, política y económica para la que nació» ${ }^{6}$. Desde esta perspectiva, pienso que sería un ejercicio beneficioso considerar las sugerencias del arquitecto y académico Juan Lund ${ }^{7}$, al afirmar que los contenidos culturales que la Universidad de Chile puede ofrecer a través de sus cuerpos estables como la Orquesta Sinfónica, el Ballet Nacional y el Coro, contribuirían a garantizar las condiciones de uso intensivo del edificio y asegurar de esta forma el contenido que una pieza arquitectónica de esta envergadura necesita proveer a la ciudadanía.

6 Iñaki Esteban. El efecto Guggenheim. Del espacio basura al ornamento. Barcelona: Editoria Anagrama, 2007.

Suplemento Artes y Letras del diario El Mercurio. Santiago, 12 de agosto de 2007
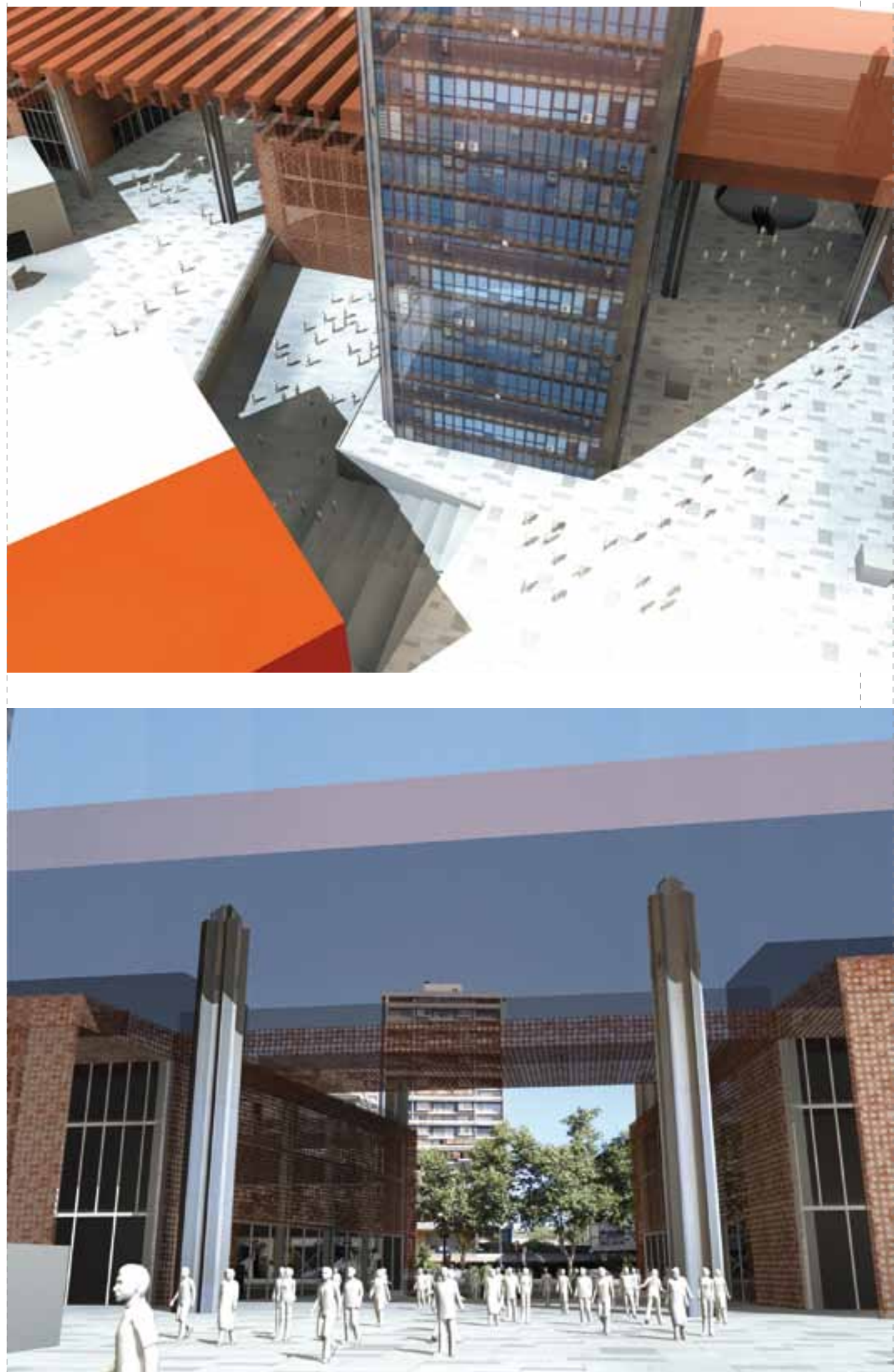